\title{
Metamodels for Composite Lattice Fuselage Design
}

\author{
Dianzi Liu, Xue Zhou, and Vassili Toropov
}

\begin{abstract}
This paper presents a novel design of an anisogrid composite aircraft fuselage by a global metamodel-based optimization approach. A 101-point design of numerical experiments (DOE) has been developed to generate a set of individual fuselage barrel designs and these designs have further been analyzed by the finite element (FE) method. Using these training data, global metamodels of all structural responses of interest have been built as explicit expressions of the design variables using a Genetic Programming approach. Finally, the parametric optimization of the fuselage barrel by genetic algorithm (GA) has been performed to obtain the best design configuration in terms of weight savings subject to stability, global stiffness and strain requirements.
\end{abstract}

Index Terms - Composite fuselage structure, anisogrid design, genetic programming, metamodel.

\section{INTRODUCTION}

In order to keep air transport competitive and safe, aircraft designers are forced for minimum weight and cost designs. Carbon composite materials combined with lattice structures for the next generation fuselage design have the potential to fulfill these requirements. This novel design of a lattice composite fuselage has been investigated recently for a new weight-efficient composite fuselage section [1].

Based on the conceptual fuselage design obtained by topology optimization with respect to weight and structural performance [2], [3], the parametric optimization of the composite lattice fuselage to obtain the optimal solution describing the lattice element geometry is performed in this paper. This detailed design process is a multi-parameter optimisation problem, for which a metamodel-based optimization technique is used to obtain the optimal lattice element geometry. Since one of the design variables, the number of helical ribs, is integer in the optimization of a lattice composite fuselage structure, a discrete form of genetic algorithm (GA) [4], [5] is used to search for the optimal solution in terms of weight savings subject to stability, global stiffness and strain requirements. Finally, the skin is interpreted as a practical composite laminate which complies with the aircraft industry lay-up rules and manufacturing requirements.

Manuscript received February 14, 2015; revised July 27, 2015. This work was supported in part by the European Commission and the Russian government within the EU FP7 Advanced Lattice Structures for Composite Airframes (ALaSCA) research project.

D. Liu is with the Faculty of Science, University of East Anglia, Norwich, NR4 7TJ, UK (e-mail: dianzi.liu@uea.ac.uk).

Xue Zhou is with the Faculty of Business, Environment and Society, Coventry University, Coventry, CV1 5FB, UK.

V. V. Toropov is with the School of Engineering and Materials Science, Queen Mary University of London, London, E1 4NS, UK.

\section{DESIGN OF EXPERIMENTS}

The quality of the metamodel strongly depends on an appropriate choice of the Design of Experiments (DOE) type and sampling size. A uniform Latin hypercube DOE based on the use of the Audze-Eglais optimality criterion [6], is proposed. The main principles in this approach are as follows: - The number of levels of factors (same for each factor) is equal to the number of experiments and for each level there is only one experiment;

- The points corresponding to the experiments are distributed as uniformly as possible in the domain of factors. There is a physical analogy of the Audze-Eglais optimality criterion with the minimum of potential energy of repulsive forces for a set of points of unit mass, if the magnitude of these repulsive forces is inversely proportional to the squared distance between the points:

$$
U=\sum_{p=1}^{P} \sum_{q=p+1}^{P} \frac{1}{L_{p q}^{2}} \rightarrow \min
$$

where $P$ is the number of points, $L_{p q}$ is the distance between the points $p$ and $q(p \neq q)$ in the system. Minimizing $U$ produces a system (DOE) where points are distributed as uniformly as possible, see Fig. 1.
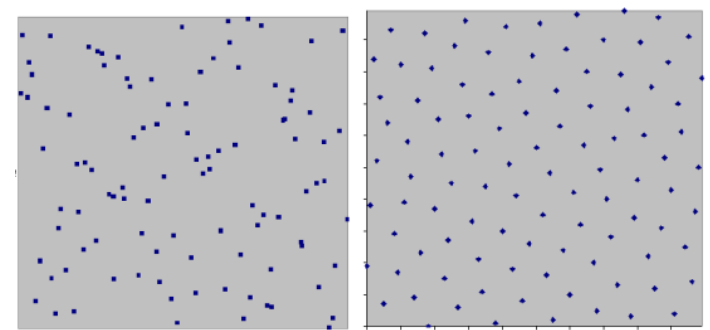

Fig. 1. Designs of experiments (100 points) generated by the conventional (left) and optimal (right) Latin hypercube technique [7].

\section{Genetic Programming (GP)}

The genetic programming code was first developed according to the guidelines provided by Koza [8], then further implemented by Armani [9]. The common genetic operations used in genetic programming are reproduction, mutation and crossover, which are performed on mathematical expressions stripped of their corresponding numerical values. Since GP methodology is a systematic way of selecting a structure of high quality global approximations, selection of individual regression components in a model results in solving a combinatorial optimization problem. In our case of design optimization, the program represents an empirical model to be used for approximation of a response function. A tree structure-based typical program, representing the 
expression $\left(x_{1} / x_{2}+x_{3}\right)^{2}$, is shown in Fig. 2.

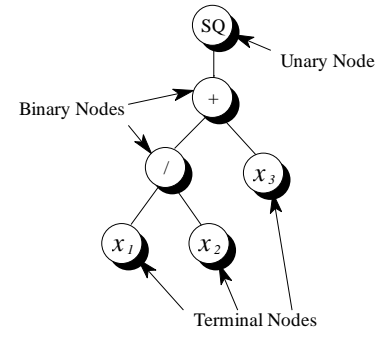

Fig. 2. Typical tree structure for $\left(x_{1} / x_{2}+x_{3}\right)^{2}$.

These randomly generated programs are general and hierarchical, varying in size and shape. GP's main goal is to solve a problem by searching highly fit computer programs in the space of all possible programs that solve the problem. This aspect is the key to find near global solutions by keeping many solutions potentially close to minima (local or global). The evolution of the programs is performed through the action of the genetic operators and the evaluation of the fitness function.

\section{Finite ElEMENT SimUlations AND MARGINS OF SAFETY}

Two FE models used in the analysis were based on a relatively coarse mesh and a much finer mesh that corresponds to a converged solution found from a mesh sensitivity study. The coarse mesh FE simulations, that are an order of magnitude faster, still reveal the most prominent features of the structural response and hence have been used in the analysis of 101 designs corresponding to the DOE points. Then, the obtained optimal solution was validated by the analysis with the fine FE mesh.

The measure of strains used were the largest strains in the structure. This consisted of the tensile and compressive strains in the frames and helical ribs, and the tensile, compressive and shears strains in the fuselage skin. These strains are normalization with respect to the maximum allowable strains in the structure. The margin of safety for strain and strength response is defined as:

$$
M S_{\varepsilon}=\frac{\varepsilon_{\max }}{\varepsilon}-1 \geq 0, \quad M S_{S}=\frac{S}{S_{\min }}-1 \geq 0, \quad M S_{B}=\lambda-1 \geq 0
$$

where $M S$ is Margin of Safety, $\varepsilon$ is the computed strain, $\varepsilon_{\max }$ is the maximum allowable strain, $S$ is the computed stiffness, $S_{\min }$ the minimum allowable stiffness, $\lambda$ is the computed linear buckling eigenvalue for the applied loads.

\section{Design VARIABles ANd Optimization of Fuselage STRUCTURE}

The ALaSCA Airframe Concept is a lattice structure with a load bearing skin and stiffeners located on either side of the skin as shown in Fig. 3. The outer stiffeners are surrounded by protective foam, which in turn is covered by a thin aerodynamic skin [2]. The optimized grid type fuselage section is a simple structure without windows or floors consisting only of the repeated structural triangular unit cell. Fig. 4 shows the finite element fuselage barrel model with the inner helical ribs in green, their counter parts on the outside of the skin in blue, the circumferential frames in yellow and the skin in red. The stiffening ribs are arranged at an angle so as to describe a helical path along the fuselage barrel skin. Hence, these ribs are called helical ribs. The helical ribs have a hat cross section, whereas the circumferential frames have a Z-shaped cross section. These ribs in conjunction with the circumferential frames create uniform triangular skin bays. The helical ribs form an angle of $2 \varphi$ between them as illustrated in Fig. 3. This angle remains constant throughout the barrel model.

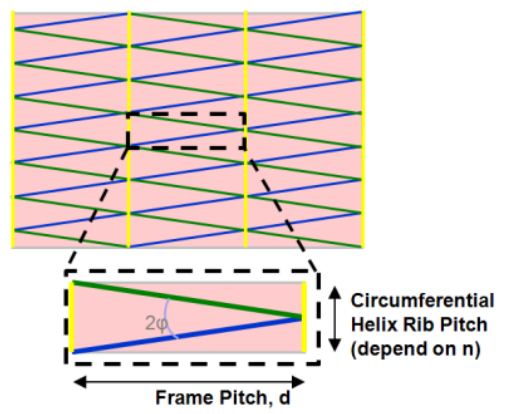

Fig. 3. Skin bay geometry.

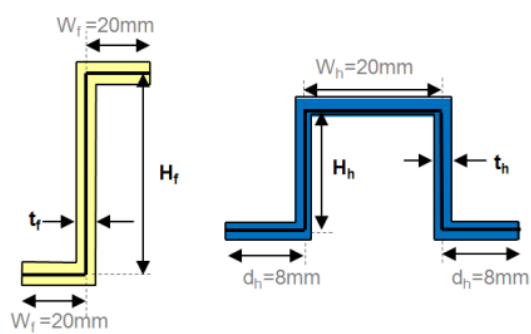

Fig. 4. Circumferential ribs and helical ribs.

The design variables are chosen to vary the geometry of the helical stiffeners and frames, the skin thickness, and the frame pitch without altering the triangular shape of the skin bay geometry. The seven optimization parameters are varied between the maximum and the minimum bounds listed in Table I. The design variables are shown in Fig. 3 and Fig. 4. The optimization constraints are strain, global stiffness and stability. The corresponding optimization responses extracted from the FE models are the largest strains (tensile and compressive strains in the frames and in the helical ribs; tensile, compressive and shear strains in the skin), the critical buckling load, and the stiffness of the fuselage. The composite material fails if it is strained beyond a maximum value. Finally, the fuselage has to have a certain stiffness in bending and in torsion to avoid excessive global deformations in flight. The design variables are varied within the bounds shown in Table I to generate fuselage structures, which are then evaluated with respect to the mentioned failure modes.

An upward gust load case at low altitude and cruise speed is applied to the modelled fuselage barrel and depicted in Fig. 5. At one end of the barrel, bending, shear, and torsion loads are applied while the opposite end is fixed. These loads are 
applied via rigid multipoint constrains, which force a rigid barrel end. While floors are not modelled, the masses from the floors are applied at the floor insertion nodes. Finally, the structural masses are applied to the skin shell elements via mass densities.

\section{RESULTS AND DISCUSSIONS}

The explicit expressions for the responses related to tensile strain, compressive strain, shear strain and weight of the fuselage barrel are built by GP. As an example, the expression for the shear strain is:

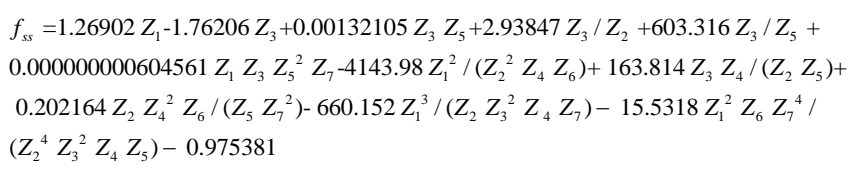

where $Z_{1}$ to $Z_{7}$ are the design variables detailed in Table I.
The parametric optimization of the fuselage barrel was performed by a Genetic Algorithm (GA) used on the GP-derived analytical metamodels. Since a GA has good non-local properties and is capable of solving problems with a mix of continuous and discrete design variables, it becomes a good choice for the fuselage barrel optimization where one of the design variables, the number of helical ribs, is integer. The results of the metamodel-based optimization and the fine mesh FE analysis are given in Table II.

TABLE I: DESIGN VARIABLES

\begin{tabular}{lcc}
\hline \hline Design variables & $\begin{array}{c}\text { Lower bound } \\
{[\mathrm{mm}]}\end{array}$ & $\begin{array}{c}\text { Upper bound } \\
{[\mathrm{mm}]}\end{array}$ \\
\hline Skin thickness $(h)$ & 0.6 & 4.0 \\
Number of helical rib pairs, $(n)$ & 50 & 150 \\
Helical rib thickness, $\left(t_{h}\right)$ & 0.6 & 3.0 \\
Helical rib height, $\left(H_{h}\right)$ & 15 & 30 \\
Frame pitch, $(d)$ & 500 & 650 \\
Frame thickness, $\left(t_{f}\right)$ & 1.0 & 4.0 \\
Frame height, $\left(H_{f}\right)$ & 50 & 150 \\
\hline \hline
\end{tabular}

TABLE II: STRUCTURAL RESPONSE VALUES FOR THE OPTIMUM DESIGN

\begin{tabular}{lllllll}
\hline \hline Response type & $\begin{array}{l}\text { Strain } \\
\text { tension }\end{array}$ & $\begin{array}{l}\text { Strain } \\
\text { compression }\end{array}$ & $\begin{array}{l}\text { Strain } \\
\text { shear }\end{array}$ & Buckling & $\begin{array}{l}\text { Torsional } \\
\text { stiffness }\end{array}$ & $\begin{array}{c}\text { Bending } \\
\text { stiffness }\end{array}$ \\
\hline Prediction by metamodel & 0.20 & 0.23 & 1.27 & 0.00 & 1.21 & 0.89 \\
Fine mesh FE analysis & 0.62 & 0.08 & 1.09 & -0.07 & 1.21 & 0.89 \\
$\begin{array}{l}\text { Composite laminate } \\
( \pm 45 / 90 / 45 / 0 /-45 / 0)_{\mathrm{s}}\end{array}$ & 1.15 & 0.19 & 1.31 & 0.13 & 1.25 & 0.29 \\
\hline \hline
\end{tabular}

TABLE III: DESIGN VARIABLE VALUES FOR THE OPTIMAL DESIGN

\begin{tabular}{|c|c|c|c|c|c|c|c|}
\hline Design variable & $\begin{array}{c}\text { Skin thickness } \\
(h), \mathrm{mm}\end{array}$ & $\begin{array}{c}\text { No. of helical } \\
\text { rib pairs, }(n)\end{array}$ & $\begin{array}{c}\text { Helical rib } \\
\text { thickness, }\left(t_{h}\right), \mathrm{mm}\end{array}$ & $\begin{array}{l}\text { Helical rib height, } \\
\left(H_{h}\right), \mathrm{mm}\end{array}$ & $\begin{array}{l}\text { Frame pitch, } \\
(d), \mathrm{mm}\end{array}$ & $\begin{array}{l}\text { Frame thickness, } \\
\left(t_{f}\right), \mathrm{mm}\end{array}$ & $\begin{array}{c}\text { Frame height, } \\
\left(H_{f}\right), \mathrm{mm}\end{array}$ \\
\hline Optimum value & 1.71 & 150.00 & 0.61 & 27.80 & 501.70 & 1.00 & 50.00 \\
\hline
\end{tabular}

Results in Table II show that buckling is the driving criterion in obtaining the optimum. The metamodel-predicted optimum has a critical margin of buckling of 0.00 with a normalized weight of 0.29 . However, when this was checked with a finite element analysis using a fine mesh, this value was found to be -0.07 that is unacceptable. This issue has to be addressed by interpreting the skin as a valid compositelaminate at the end of this Section.

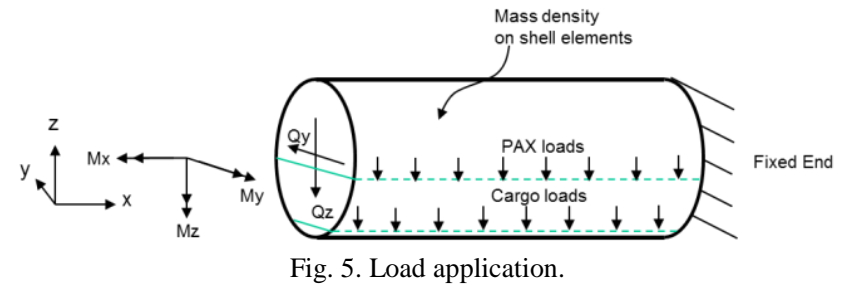

The predicted tensile strain margin of 0.20 is conservative when compared the 0.62 margin obtained by the FE analysis. The predicted compressive and shear strain of 0.23 and 1.27 , respectively, are not conservative compared to the compressive strain margin of 0.08 and the shear margin of 1.09 obtained by the FE analysis. This is acceptable as these are not the critical margins. The predicted stiffness margins are the same as the margins obtained by the FE analysis but do not act as critical constraints in this design optimization problem. The design variable set for the final optimum geometry is listed in Table III. The length of the frame pitch is
$501.7 \mathrm{~mm}$ which is close to the lower bound of 500. The resulting small triangular skin bays have a base width of 83.78 $\mathrm{mm}$, a height of $501.7 \mathrm{~mm}$ and a small angle between the crossing helical ribs of $2 \varphi=9.55^{\circ}$. Such small and skinny-triangular skin bays are excellent against buckling. There is a good correspondence of the obtained results with the analytical estimates of DLR that produced the value of $2 \varphi=12^{\circ}[10]$.

Since the optimal design only used smeared ply properties, the skin thicknesses had to be corrected to account for a standard CFRP ply thickness of $0.125 \mathrm{~mm}$. This means that the skin thickness is increased from $1.71 \mathrm{~mm}$ to $1.75 \mathrm{~mm}$ and plies of $0^{\circ}, 45^{\circ},-45^{\circ}$ and $90^{\circ}$ orientation arranged in a balanced and symmetric laminate have to be used to comply with the aircraft industry lay-up rules and manufacturing requirements [11]-[13]. The structural responses obtained by the FE analysis with the $( \pm 45 / 90 / 45 / 0 /-45 / 0)_{\mathrm{s}}$ laminate skin are given in Table II.

Incorporating the ply thicknesses into the design has increased the buckling margin of safety making all margins positive. Therefore a light-weight design which fulfils the stability, global stiffness and strain requirements has been obtained.

\section{CONCLUSION}

Parametric optimization was applied to the detailed design 
of a fuselage barrel section by using Genetic Algorithms on a metamodel generated with Genetic Programming. The optimum structure was obtained by performing parametric optimization subject to stability, global stiffness and strain requirements, then its optimal solution and structural responses were verified by finite element simulations. The stability criterion is the driving factor for the skin bay size and the fuselage weight. By interpreting the skin modelled with smeared ply properties as a real-life composite laminate, a practical lay-up with a standard ply thickness of $0.125 \mathrm{~mm}$ has been obtained as $( \pm 45 / 90 / 45 / 0 /-45 / 0)_{s}$. It is concluded that the use of the global metamodel-based approach has allowed to solve this optimization problem with sufficient accuracy as well as provided the designers with a wealth of information on the structural behaviour of the novel anisogrid composite fuselage design.

\section{REFERENCES}

[1] V. V. Vasiliev, "Anisogrid composite lattice structures Development and aerospace applications," Composite Structures, no. 94, pp. 1117-1127, 2012.

[2] S. Niemann, B. Kolesnikov, H. Lohse-Busch, C. Hühne, O. M. Querin, D. Liu, and V. V. Toropov, "Conceptual design of an innovative lattice composite fuselage using topology optimization," Aeronautical Journal, vol. 117, no. 1197, November 2013.

[3] ALaSCA (Advanced Lattice Structures for Composite Airframes) EU FP7 Project. (2010). [Online]. Available: http://cordis.europa.eu/projects/index.cfm?fuseaction=app.details\&R $\mathrm{EF}=97744$

[4] Z. Michalewicz, Genetic Algorithms + Data Structures = Evolution Programs, Springer-Verlag, 1992.

[5] S. J. Bates, J. Sienz, and V. V. Toropov, "Formulation of the optimal latin hypercube design of experiments using a permutation genetic Algorithm," presented at 45th AIAA/ASME/ASCE/AHS/ASC Structures, Structural Dynamics, and Materials Conference, Palm Springs, CA, April 19-22, 2004.

[6] P. Audze and V. Eglais, "New approach for planning out of experiments," Problems of Dynamics and Strengths, vol. 35, pp. 104-107, Zinatne Publishing House, Riga, 1977.
[7] V. V. Toropov, U. Schramm, A. Sahai, R. D. Jones, and T. Zeguer, "Design optimization and stochastic analysis based on the moving least squares method," presented at 6th World Congresses of Structural and Multidisciplinary Optimization, Rio de Janeiro, 2005.

[8] J. R. Koza, Genetic Programming: On the Programming of Computers by Means of Natural Selection, Cambridge, USA: MIT Press, 1992.

[9] U. Armani, "Development of a hybrid genetic programming technique for computationally expensive optimisation problems," $\mathrm{Ph} . \mathrm{D}$. dissertation, School of Civil Engineering, University of Leeds, Leeds, UK, 2014.

[10] H. Lohse-Busch, C. Hühne, D. Liu, V. V. Toropov, and U. Armani, "Parametric optimization of a lattice aircraft fuselage barrel using metamodels built with genetic programming," in Proc. the Fourteenth International Conference on Civil, Structural and Environmental Engineering Computing, Stirlingshire, UK: Civil-Comp Press, 2013.

[11] M. C. Y. Niu, Composite Airframe Structures, Practical Design Information and Data, Hong Kong: Conmilit Press Ltd., 1992.

[12] D. Liu, V. V. Toropov, O. M. Querin, and D. C. Barton, "Bilevel optimization of blended composite wing panels," Journal of Aircraft, vol. 48, pp. 107-118, 2011.

[13] C. Kassapoglou, Design and Analysis of Composite Structures: With Applications to Aerospace Structures, 2nd Edition, John Wiley \& Sons, 2013.

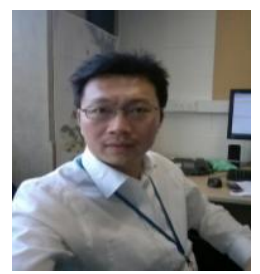

Dianzi Liu was born in China and obtained his $\mathrm{PhD}$ in mechanical engineering in the University of Leeds, UK in 2010. His PhD research project was bi-level optimization of composite aircraft wing panels subject to manufacturing constraints.

He has been a lecturer in engineering in the University of East Anglia, Norwich, UK since 2014. Before joining the University, he spent three years in the University of Leeds as a research fellow. His research interests focus on composite structures, structural analysis, simulation and optimization driven designs, implementation and application of optimization algorithms/techniques in the mechanical, manufacturing and aerospace engineering.

Dr. Liu is a member of South Asia Institute of Science and Engineering (SAISE), a member of American Institute of Aeronautics and Astronautics (AIAA) and a member of International Society for Structural and Multidisciplinary Optimization (ISSMO). Dr. Liu was awarded the runner-up prize for his research paper in the ISSMO-Springer Prize competition in 2009. 